\title{
Pro-Inflammatory Cytokines and Gelatinases in Climatic Droplet Keratopathy
}

\author{
Juba M. Holopainen, ${ }^{1}$ Alexandra Robciuc, ${ }^{1,2}$ Thamara A. Cafaro, ${ }^{4}$ Maria F. Suarez, ${ }^{4}$ \\ Yrjö T. Konttinen, ${ }^{3}$ Hind M. Alkatan, ${ }^{5}$ Khalid F. Tabbara, ${ }^{5}$ Taina Tervahartiala, ${ }^{6}$ Timo Sorsa, ${ }^{6}$ \\ Julio A. Urrets-Zavalia, ${ }^{7}$ and Horacio M. Serra ${ }^{4}$
}

Purpose. Climatic droplet keratopathy (CDK) is a degenerative disease of the cornea with possible involvement from matrix metalloproteinases (MMPs). Therefore, the authors investigated histologic distribution, levels, and molecular forms of MMP2 and MMP-9, as well as tear fluid levels of MMPs and cytokines in CDK patients. They additionally examined UV-B-irradiation effect on production of gelatinases and cytokines by human corneal epithelial (HCE) cell culture model.

Methods. Tears were collected from 20 unrelated individuals (10 with CDK and 10 controls). CDK affected corneas were haematoxylin-eosin stained and the presence and distribution of MMP-2 and MMP-9 was examined using immunohistochemistry. Gelatinases and cytokine secretion was measured in tears and supernatants from UV-B-exposed HCEs by immunoblotting, gelatin zymography, and protein array, respectively.

Results. MMP-2 and MMP-9 values were significantly higher in tears collected from CDK patients than healthy controls and were accompanied by pro-inflammatory cytokine secretion. Immunohistochemistry showed that MMP-2 was expressed at the basement membrane zone in both control and affected corneas, but also marked the edges of the granular CDK deposits; MMP-9 expression was restrained to basal layers of the epithelium and was markedly induced in CDK corneas. In

From the ${ }^{1}$ Helsinki Eye Lab, Department of Ophthalmology, University of Helsinki, Helsinki, Finland; the ${ }^{2}$ Public Health Genomic Unit, National Institute for Health and Welfare and Institute for Molecular Medicine Finland, Helsinki, Finland; the ${ }^{3}$ Department of Medicine, Helsinki University Central Hospital, ORTON Orthopaedic Hospital of the ORTON Foundation, Helsinki, Finland; the ${ }^{4}$ Center for Research in Clinical Biochemistry and Immunology, Department of Clinical Biochemistry, National University of Córdoba, Córdoba, Argentina; ${ }^{5}$ The Eye Center and The Eye Foundation for Research in Ophthalmology, Riyadh, Saudi Arabia; the ${ }^{6}$ Department of Oral and Maxillofacial Diseases, Helsinki University Central Hospital, Institute of Dentistry, University of Helsinki, Helsinki, Finland; and the ${ }^{7}$ Department of Ophthalmology, University Clinic Reina Fabiola, Catholic University of Córdoba, Córdoba, Argentina.

Supported by grants from the Sigrid Juselius Foundation (JMH), the Academy of Finland (JMH, TS), the Finnish Eye Foundation (JMH), the Helsinki University Central Hospital Research Foundation (JMH, TS), the Fund for the Scientific and Technological Research (PICT 38052), and the National Council of Scientific and Technical Research (PIP 11220080101455 ).

Submitted for publication January 6, 2011; revised March 7 and April 16, 2012; accepted April 26, 2012.

Disclosure: J.M. Holopainen, None; A. Robciuc, None; T.A. Cafaro, None; M.F. Suarez, None; Y.T. Konttinen, None; H.M. Alkatan, None; K.F. Tabbara, None; T. Tervahartiala, None; T. Sorsa, None; J.A. Urrets-Zavalia, None; H.M. Serra, None

Corresponding author: Juha M. Holopainen, Helsinki Eye Lab, Department of Ophthalmology, University of Helsinki, Haartmaninkatu 4 C, 00290 Helsinki, Finland; juha.holopainen@hus.fi.
HCE cells, UV-B increased gelatinase secretion, with a striking effect on MMP-9, and was preceded by pro-inflammatory cytokine release.

Conclusions. The authors demonstrate that the corneal epithelium could participate in CDK development as a source of cytokines and gelatinases. Additionally, in HCE cells, UV-Bmodulated cytokine and subsequent MMP secretion. Local inhibition of cytokine secretion and gelatinases may prevent CDK progression. (Invest Ophthalmol Vis Sci. 2012;53:35273535) DOI:10.1167/iovs.12-9441

$\mathrm{C}$ limatic droplet keratopathy (CDK) is a degenerative corneal disease related to advanced age and multiple environmental factors such as corneal microtraumas, low humidity, and lack of adequate protection for exposure to UV light. CDK most commonly affects males over 40 years old, in the form of progressive opacities of the anterior layers of the cornea in both eyes. CDK is rare in temperate latitudes, but it is commonly linked to overexposure to UV irradiation and is endemic in some areas, with a high proportion of the population over 40 years old being affected. ${ }^{1}$

Histopathologically globular deposits of different sizes are observed by light microscopy, under the corneal epithelium, within the Bowman's layer and the anterior stroma. Commonly, signs of disruption of the Bowman's membrane and scarring, as well as epithelial thinning and elevation, are seen in advanced stages of the disease. The origin and the exact nature of the globular droplets remain unknown; however, the authors' recent proteome analysis suggested that they were composed of fibril/aggregation forming proteins. ${ }^{2}$ Yet, the authors were not able to provide a pathogenic model for CDK, nor were they able to identify those proteins that were responsible for the breakdown of the highly organized corneal structure.

Early findings showed the disruption of Bowman's membrane, prompting the authors to study the role of matrix metalloproteinases (MMPs) in CDK. Under normal conditions, MMPs are expressed at relatively low levels and their tight regulation gives way to upregulation only when degradation is required. ${ }^{3}$ The authors recently reported that in human reflex tears collected from CDK patients, the levels of gelatinases were increased, while their tissue inhibitors (TIMPs) were decreased. ${ }^{4}$ These findings suggested a role for MMPs in the disruption of the corneal architecture resulting in loss of corneal transparency. The authors now further explored the role of gelatinases (MMP-2 and MMP-9) and cytokines in the development of CDK by analyzing the tissue distribution of gelatinases in human corneas and also human nonreflex tears. In addition, they performed in vitro experiments with UV-B irradiation of cultured human corneal epithelial (HCE) cells to establish if UV radiation could be the underlying factor for the increased proteolysis and inflammation. The results suggest 
that in CDK the corneal epithelium may have a crucial responsibility as UV stress elicits increased expression of inflammatory cytokines and proteolytic enzymes.

\section{MATerials ANd Methods}

\section{Individuals}

Ten patients (nine males and one female; age $67.4 \pm 10.2$; range 58-76 years) and 10 controls (nine males and one female; age 63.6 \pm 10.7 ; range 57-71 years) living in an area of the northwest Patagonia region in Argentina were enrolled into this study. CDK patients and controls had a complete eye examination as described previously. ${ }^{5}$ Altogether 15 to $20 \mu \mathrm{L}$ of tear fluid from both eyes was collected with minimal conjunctival irritation, without any chemical or physical stimulation, using disposable microcapillaries and rapidly frozen at $-80^{\circ} \mathrm{C}$ until analysis. ${ }^{4}$ Informed consent was obtained from all participants, and the study was approved by the institutional review board of the Clínica Universitaria Reina Fabiola. The study was conducted in accordance with the tenets of the Declaration of Helsinki.

\section{Assessment of Gelatinases (MMP-2 and MMP-9) in Human Tear Samples}

The volumes of tear samples were adjusted to obtain an equal amount of protein for zymographic analysis as described previously. ${ }^{4}$ To measure the activity of gelatinases from human tear samples, $30 \mu \mathrm{g}$ of tear proteins were electrophoresed in nonreducing conditions through $7.5 \%$ polyacrylamide gels copolymerized with $1.5 \%$ gelatin as the substrate (gelatin SDS-PAGE). ${ }^{4,6}$ The presence of proteinases in gels was quantified using image analysis software (Gel-Pro Analyzer 3.1; Media Cybernetics, Inc., Silver Spring, MD). Whole blood gelatinases were used as positive controls and MMP-2 and MMP-9 were identified by molecular size using molecular mass standards (Bio-Rad, Hercules, CA). For cell supernatants, zymographies were performed using $14 \mu \mathrm{L} /$ sample electrophoresed on $10 \%$ SDS-PAGE containing $1 \mathrm{mg} / \mathrm{mL}$ gelatin labeled with 2-methoxy-2,4dephenyl- $3(2 \mathrm{H})$ furanone. ${ }^{7}$ Next, gels were washed in $2.5 \%$ Triton $\mathrm{X}$ $100,0.02 \mathrm{NaN}_{3}$, and then incubated in the activation solution $(50 \mathrm{mM}$ Tris, $5 \mathrm{mM} \mathrm{CaCl}_{2}, 1 \mu \mathrm{M} \mathrm{ZnCl}_{2}, 0.02 \mathrm{NaN}_{3}, \mathrm{pH} 7.5$ ) at $37^{\circ} \mathrm{C}$ for 1 to 5 days. The degradation of gelatin was followed and photographed using a longwave UV light (UVIDOC BTS-20.M; Uvitec Ltd., Cambridge, UK).

\section{Haematoxylin and Eosin (H-E) Staining of Corneal Samples}

Corneal tissue from four CDK patients and three healthy subjects were fixed in $10 \%$ buffered formaldehyde (pH 6.8) and embedded in paraffin. All corneal tissue was sectioned to $5 \mu \mathrm{m}$ sections perpendicular to the epithelial surface. Some were stained in haematoxylin (for 3 minutes, differentiated with three dips in $0.5 \%$ hydrochloric acid in $70 \%$ ethanol, and blued with $2 \% \mathrm{NaHCO}_{3}$ for 1 minute) and counterstained with $1 \%$ aqueous eosin (BDH Chemical Ltd., Poole, UK). Sections were examined and photographed with a microscope (Nikon Eclipse TE2000-E; Nikon Corp., Tokyo, Japan) equipped with a camera (Nikon Digital Sight DS-5MC; Nikon Corp.).

\section{Immunohistochemical Staining of MMP-2 and MMP-9 in Normal and CDK Corneas}

Formalin-fixed, paraffin-embedded 5 - $\mu$ m-thick corneal sections from four patients with CDK and three controls were used for immunohistochemical localization of MMP-2 and MMP-9. Avidin-biotin peroxidase complex staining (VectaStain, Burlingame, CA), together with the affinity purified goat anti-human MMP-2 IgG (R\&D Systems, Minneapolis, MN) or polyclonal affinity purified goat anti-human MMP-9 IgG (R\&D Systems), was used according to manufacturer's instructions. ${ }^{8}$ Normal goat, rabbit, or mouse IgG with an irrelevant specificity, but of the same subclass and concentration as the primary antibody or omission of the primary antibody, were used as staining controls. Counterstaining with haematoxylin was followed by mounting in Mountex medium (Histolab Products, Gothenburg, Sweden).

\section{Cytokine/Chemokine Beadarray}

Five microliters of human tear fluid was used for cytokine/chemokine analysis using the human cytokine/chemokine Multiplex assay kit (BioRad Laboratories, Richmond, CA). This ELISA and flow cytometrybased technique detects IL-1 $\beta$, IL-2, IL-4, IL-5, IL-6, IL-7, IL-8, IL-10, IL12, IL-13, IL-17, G-CSF, GM-CSF, IFN- $\gamma$, monocyte chemotactic protein 1 (MCP-1), macrophage inflammatory protein-1 $\beta$ (MIP-1 $\beta$ ), and TNF- $\alpha$, and was used according to the manufacturer's instructions.

\section{In Vitro Experiments}

Simian Virus 40 (SV 40)-immortalized HCE cells were cultured in Dulbecco's modified Eagle's medium: Nutrient Mixture F-12 (DMEM/ F12), supplemented with $15 \%$ fetal bovine serum, $5 \mu \mathrm{g} / \mathrm{mL}$ insulin, 10 $\mathrm{ng} / \mathrm{mL}$ human EGF, $1 \mu \mathrm{g} / \mathrm{mL}$ glutamine, $40 \mu \mathrm{g} / \mathrm{mL}$ gentamycin (all from Invitrogen Corp., Carlsbad, CA), and $0.1 \mu \mathrm{g} / \mathrm{mL}$ cholera toxin (Sigma, St. Louis, MO). The cells were subcultured twice a week. Confluent corneal epithelial cells were washed once with warm PBS. The PBScovered monolayer was exposed to UV-B radiation in open culture vessels with energy from 0 to $10 \mathrm{~mJ} / \mathrm{cm}^{2}$ in a microprocessor controlled UV crosslinker (Spectroline XL-1500; Spectronics Co., Westbury, NY). After the UV-B stimulation the cells were incubated for 16,24 , or 48 hours. Conditioned medium was collected and centrifuged for 10 minutes at $400 \mathrm{~g}$ to eliminate floating cells and debris. Actin immunoblotting was performed to ensure that the media had no cellular contamination. The cellular monolayer was washed with cold PBS and lysed on ice for total protein concentration measurement. All data were normalized to cell lysate protein concentration.

\section{Western Blotting}

Cell supernatant samples $(200 \mu \mathrm{L}$ filtrated dry and redissolved in Laemmli buffer) and molecular weight standards were run on a $4 \%$ to $12 \%$ Bis-Tris NuPAGE gel (Invitrogen Corp.) for 2 hours at $140 \mathrm{~V}$. Secreted proteins were then electrically transferred to a nitrocellulose membrane ( $400 \mathrm{~mA}$ for 45 minutes). MMPs and TIMP-1 were detected separately using specific antibodies ${ }^{4,6}$ and an electrochemiluminescence detection kit (GE Healthcare, Amersham, UK). Secondary goat anti-rabbit antibody conjugated with horseradish peroxidase was from DAKO Immunoglobulins (Dako, Glostrup, Denmark).

\section{MMP-2 and MMP-9 ELISA}

To measure MMP-2 and MMP-9 levels in the HCE-conditioned medium, two commercially available ELISA kits were used following the instructions provided by the manufacturers. MMP-2 ELISA (Biotrak ELISA system; GE Healthcare) was designed to specifically recognize MMP-2 as pro-enzymes and enzyme in complexes with TIMPs, but not active forms; while the MMP-9 ELISA (Quantikine ELISA, Human MMP-9 Immunoassay; R\&D Systems Europe, Ltd., Abingdon, UK) measures the $92-\mathrm{kDa}$ pro- and $82-\mathrm{kDa}$ active forms, but not the $65-\mathrm{kDa}$ form. The reactions were carried out in 96-well plates and analyzed at $450 \mathrm{~nm}$. Cell-free supernatant was used at a one-to-one dilution in assay buffer. Calculated values were normalized to protein concentration from cellular lysate and reported as nanogram per milligram of protein.

\section{Assessment of Secreted Cytokines from HCE Cell Culture}

For parallel determination of relative levels of secreted chemokines and cytokines, the authors used a Proteome Profiler Array - Human Cytokine Array Panel A (R\&D Systems Inc., Minneapolis, MN). The 


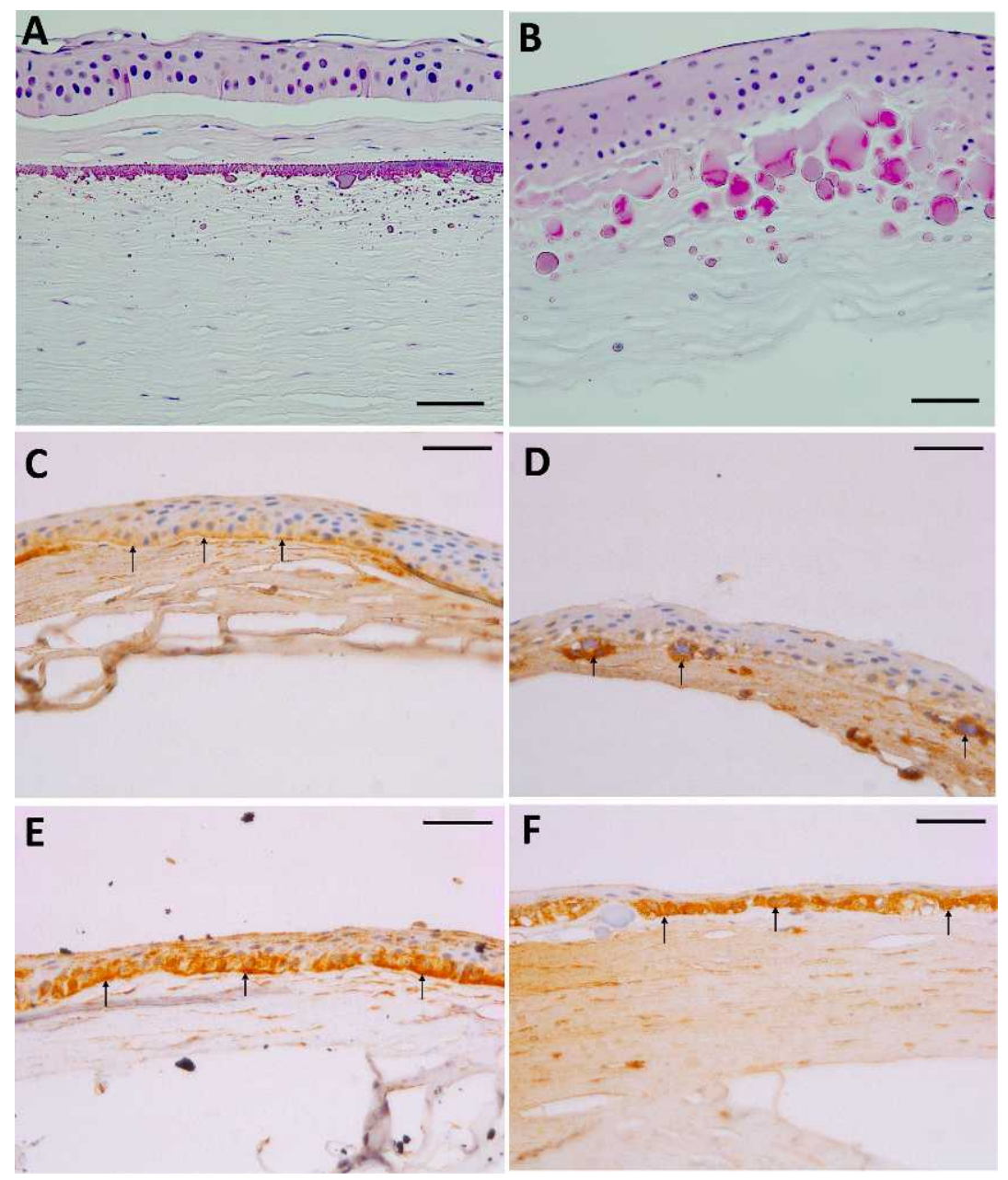

Figure 1. Histology, MMP-2 and MMP-9 in unaffected and CDK corneas. (A) Early stage CDK cornea shows a thinned corneal epithelium, intact Bowman's layer, and regularly organized corneal stroma, but multiple droplets in the anterior stroma. (B) Later stage CDK cornea shows multiple droplets of varying shape and size in the anterior stroma, epithelium is damaged, and the Bowman's layer is dissolved. MMP-2 expression is observed in the basement membrane area in both control (C) and CDK (D) corneas. MMP-2 also accumulates around the corneal deposits (arrows) in corneas from CDK patients. In healthy corneas (E), weak MMP-9 staining is observed in the basal cells and the basement membrane area, whereas in CDK corneas (F) strong MMP-9 staining is seen in the basal corneal epithelial cells (arrows). Scale bar in images represents $50 \mu \mathrm{m}$.

array measures levels of 36 different chemokines and cytokines (spotted in duplicates on a nitrocellulose paper) in the same sample. Samples were prepared following the manufacturer's instructions. Briefly, conditioned media from confluent cells was cleared from floating cells and debris, and an aliquot of $700 \mu \mathrm{L}$ was mixed with the kit reagents and assayed immediately.

\section{Analysis}

The zymography and the Western blot data were evaluated by densitometry analysis using the ImageJ software (National Institutes of Health [NIH] free software v1.42q software; National Institutes of Health, Bethesda, MD) on nonaltered TIFF images of the gels. Data were presented as mean values from three independent experiments \pm SD, unless otherwise specified. The results were evaluated by the MannWhitney $U$ test. A $P$ value less than 0.05 was considered significant.

\section{RESULTS}

\section{Light Microscopy of Corneas Affected by CDK}

The superficial corneal epithelium evaluated by H-E staining was normal or nearly normal in early stages of CDK (Fig.
TABLE Multiplex ELISA of Cytokines and Chemokines in Human Tear Fluid from CDK Patients and Healthy Controls

\begin{tabular}{lccc}
\hline \multicolumn{4}{c}{ Cytokine and Chemokine Proteins } \\
\hline & CDK & Controls & $\boldsymbol{P}$ \\
\hline IL-1 $\beta$ & $88.4 \pm 64.4$ & $14.3 \pm 5.8$ & 0.0027 \\
IL-8 & $4427.0 \pm 1574.0$ & $1882.0 \pm 1283.0$ & 0.0007 \\
IL-5 & $15.0 \pm 5.7$ & $9.9 \pm 11.0$ & 0.2417 \\
IL-6 & $363.3 \pm 301.7$ & $126.0 \pm 109.5$ & 0.159 \\
IL-7 & $1901.0 \pm 1024.0$ & $985.0 \pm 71.0$ & 0.1064 \\
TNF- $\alpha$ & $46.3 \pm 61.2$ & 156.0 & NA \\
MCP-1 & $2354 \pm 1378$ & $1164 \pm 306$ & 0.1205 \\
MIP-1b & $1270 \pm 664$ & $804 \pm 207$ & 0.2046 \\
G-CSF & $217 \pm 197$ & $192 \pm 169$ & 0.8133 \\
IL-13 & $12.7 \pm 8.3$ & $9.4 \pm 8.3$ & 0.6847 \\
IL-2 & 0 & 0 & NA \\
IL-17 & 0 & 0 & NA \\
IL-12 & $9.3 \pm 3.7$ & 0 & NA \\
GM-CSF & $68.8 \pm 148.6$ & 0 & NA \\
IL-4 & 0 & $5.9 \pm 3.8$ & NA \\
IL-10 & 2.9 & $9.8 \pm 13.6$ & NA \\
INF- $\gamma$ & $622.1 \pm 149.9$ & 232.7 & NA \\
\hline
\end{tabular}




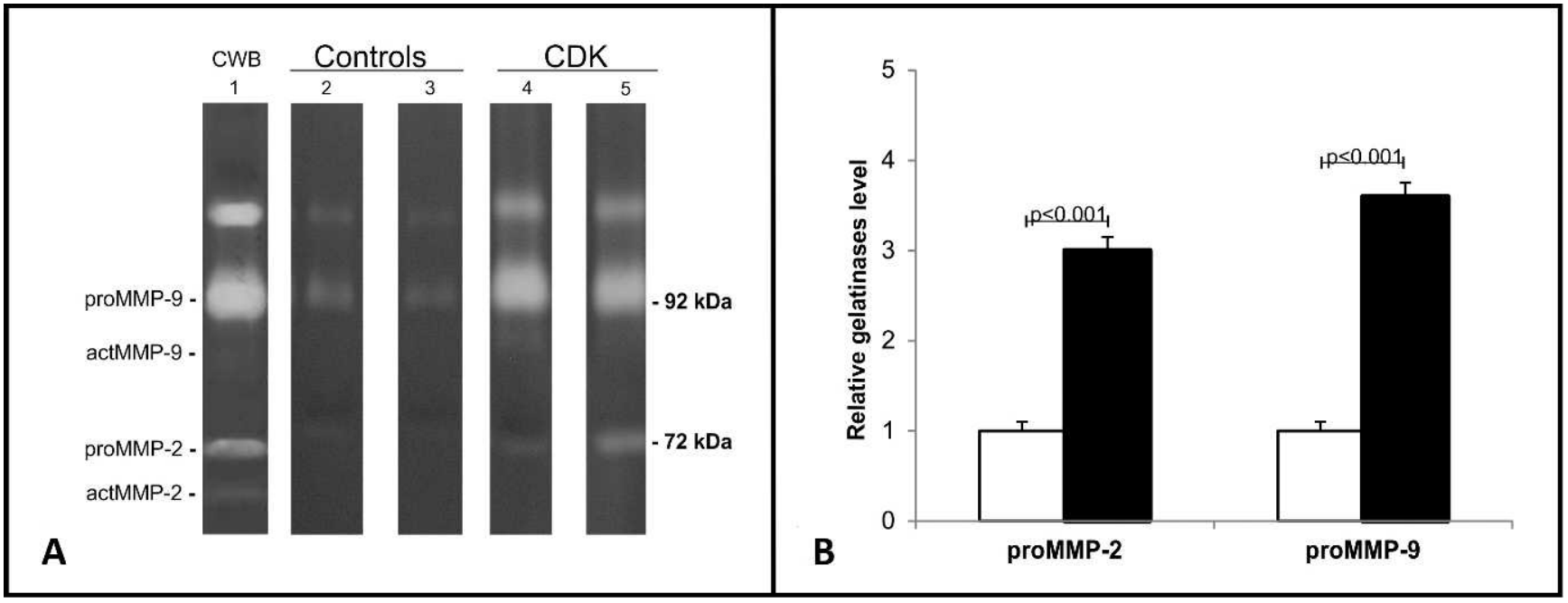

Figure 2. Tear fluid gelatinases from CDK patients and controls. (A) Representative zymography of the tear samples. Lane 1 corresponds to capillary whole blood gelatinases (CWB) used as a positive control. Gelatinolytic bands of 92 and $72 \mathrm{kDa}$ corresponding to pro-MMP-9 and proMMP-2, respectively, as well as active MMP-9 and MMP-2 are marked on the left margin. Lanes 2 to 3 control tears and lanes 4 to 5 CDK tears. (B) The levels of pro-MMP-2 and pro-MMP-9 from healthy controls (white bars) was set to 1 and then the relative difference was calculated by dividing the levels of pro-MMP-2 and pro-MMP-9 from CDK patients (black bars) by the levels of healthy controls.

1A), whereas it was thinned, elevated, and disturbed in later stages of CDK (Fig. 1B). Bowman's layer was clearly damaged in some samples. In all cases, the superficial corneal stroma contained granules of variable size and shape (Figs. 1A, 1B), and the number of these granules correlated positively with the stage of the disease. The posterior stroma, as well as the endothelium, appeared unremarkable (not shown).

\section{Immunohistochemical Detection of MMP-2 and MMP-9 in Human Corneas Affected by CDK}

MMP-2 was expressed mostly at the basement membrane zone of the corneal epithelium in both control and CDK specimens (Figs. 1C, 1D). In most CDK corneas, the edges of the granular deposits were strongly positively stained for MMP-2 (Fig. 1D), but the core of the granules did not stain for
MMP-2. Normal corneas showed only weak MMP-9 staining (Fig. 1E). However, in CDK specimens corneal epithelial basal cells were strongly positive for MMP-9 (Fig. 1F). The granules, or the edges of the granules, did not show MMP-9 immunoreactivity.

\section{Detection and Characterization of Gelatinases (MMP-2 and MMP-9) in Tear Fluid of CDK Patients and Healthy Subjects}

Figure 2A shows a representative zymogram, in which gelatinolytic bands of 72 (pro-MMP-2) and $92 \mathrm{kDa}$ (proMMP-9) were detected in CDK patients and controls. The active forms of MMP-2 or MMP-9 were not observed in either group. The mean MMP-2 activity was 3-fold higher in patients relative to controls $(95.3 \pm 10.5$ vs. $31.0 \pm 4.5$ arbitrary units [AU]; $P<0.001$ ), whereas the activity of latent-MMP-9 was
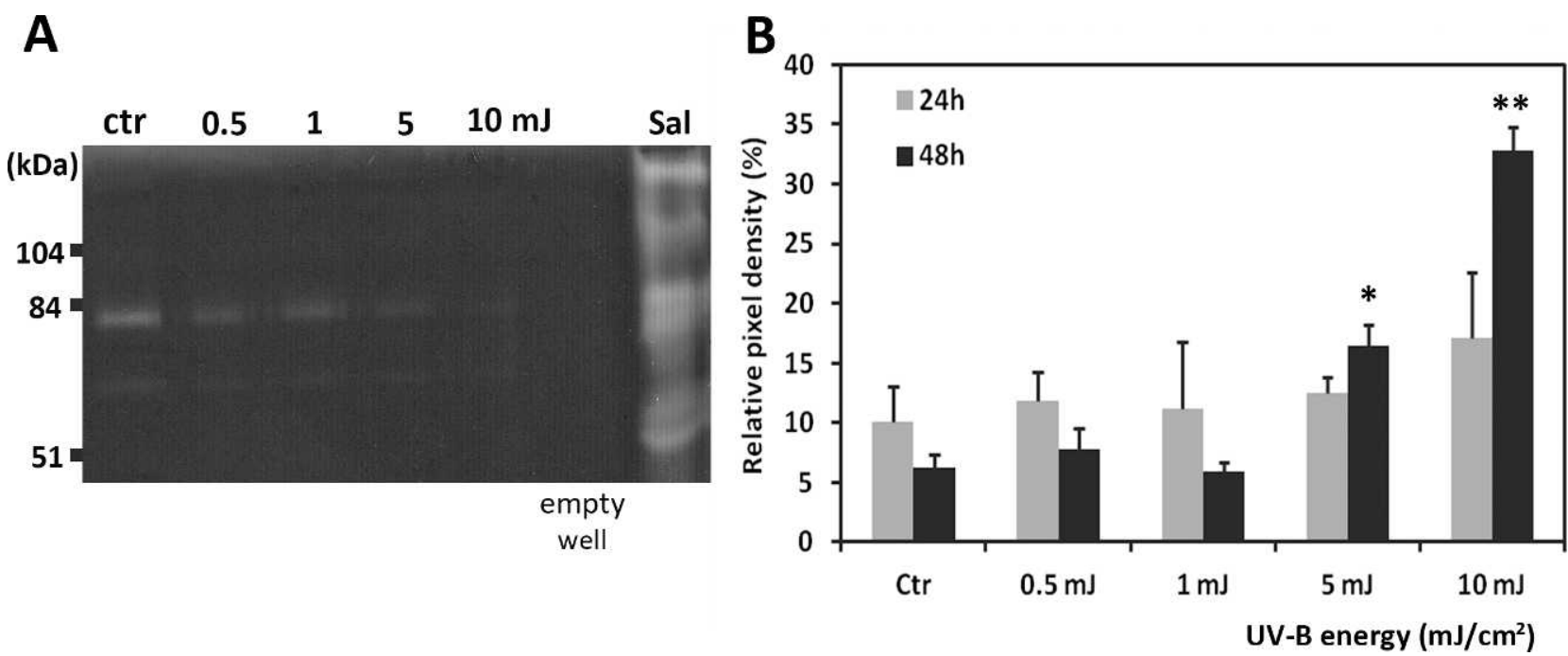

FIGURE 3. Gelatin zymography of UV-exposed HCE conditioned media. (A) Representative zymography result at 24 hours poststimulation; (B) densitometry analysis. Equal amounts of media were loaded on the gelatin SDS-PAGE. Densitometry units were normalized to protein concentration from cell lysates. ${ }^{*} P<0.05$ compared to control; ${ }^{* *} P<0.01$ compared to control. 


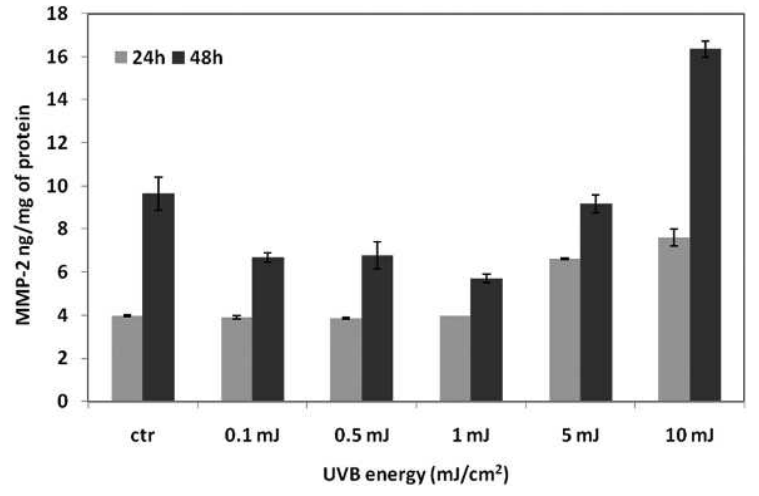

Figure 4. ELISA measurement of MMP-2 levels in media from UV stimulated cells at 24 and 48 hours after irradiation. Data were normalized to protein concentration from total cell lysates.

A
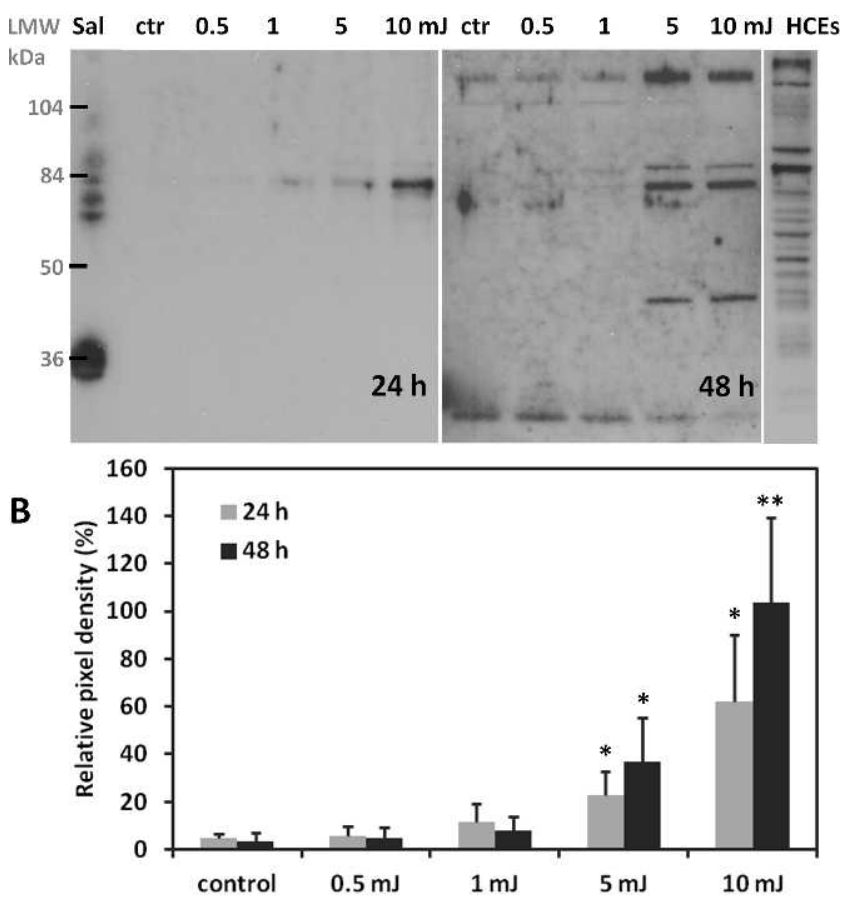

C

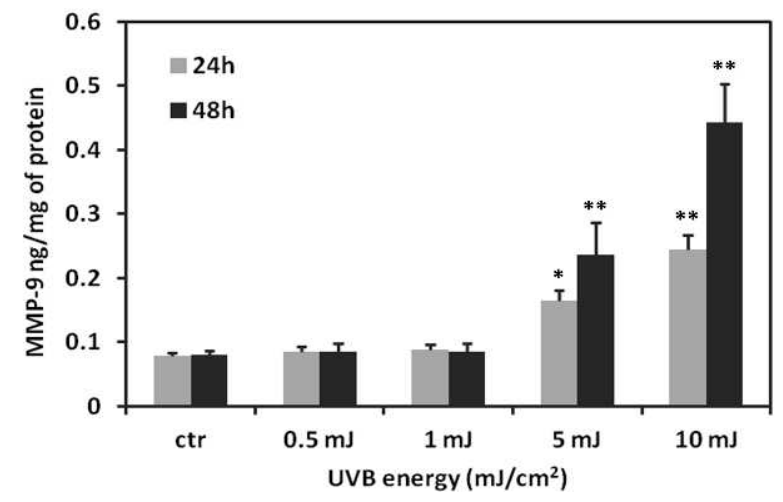

Figure 5. MMP-9 in HCE media following UV-B irradiation. (A) Representative Western blot image; (B) densitometry analysis. Equal amounts of media were loaded on a $4 \%$ to $12 \%$ Bis-Tris NuPAGE gel. Data is presented as relative densitometry units normalized to protein concentration. Low molecular weight ladder (LMW); control (ctr); saliva sample as positive control (Sal); human corneal epithelial cells lysate; (C) ELISA measurement of MMP-9 in HCE media (the immunologic assay measures pro-MMP-9 as well as active MMP-9). ${ }^{*} P$ $<0.05$ compared to control; ${ }^{* *} P<0.01$ compared to control.

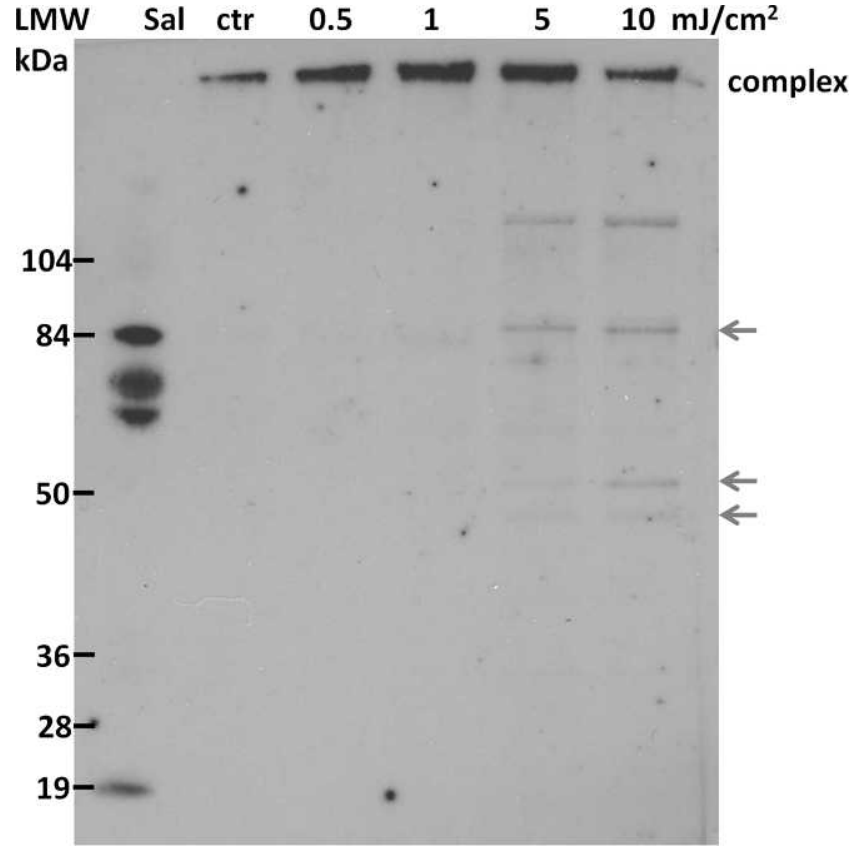

Figure 6. UV-induced secretion of MMP-8 isoforms. Only data from 48 hours after UV stimulation are shown. Bands in the molecular weight range of 75 to $80 \mathrm{kDa}$ represent the inactive form of the PMN type of MMP-8, while bands in the 40 to $55 \mathrm{kDa}$ are fibroblast-type MMP-8 enzyme forms. A saliva sample (Sal) was used as positive control.

nearly 4-fold higher in patients compared to the controls (1882 \pm 214 vs. $519.3 \pm 91.5$ AU; $P<0.001$ ) (Fig. $2 \mathrm{~B}$ ).

\section{Multiplex Protein Array Analysis of Normal and CDK Tears}

Multiplex ELISA with a panel of 17 cytokine and chemokine proteins was used to analyze the tear fluid obtained from healthy controls and CDK patients. IL-1 $\beta$, IL-5, IL-6, IL-7, IL-8, MCP-1, and MIP-1 $\beta$ were detected in all CDK tear samples, whereas in controls, IL-1 $\beta$ and IL-8 were the only cytokines found in all samples analyzed. As shown in the Table, proinflammatory IL-1 $\beta$ and IL-8 levels were significantly higher in CDK patients than in the control subjects. It is worth noting that TNF- $\alpha$ was detected at measurable levels in 6 of 10 CDK patients and in only 1 of 10 control subjects. IL-2 and IL-17 were not detected in any sample; IL-12 and GM-CSF were not detected in any control tear sample. IL-4, IL-10, and IL-13, as well as IFN- $\gamma$, were detected in low quantities or in just a few samples.

\section{In Vitro Exposure of HCE Cells to UV-B and MMPs/ TIMPs Expression}

Animal models of CDK are lacking; therefore, in order to gain mechanistic insight in the development of CDK, the authors used a cell culture model of HCE cells. Gelatin zymographic of HCE conditioned media showed measurable gelatin lysis only after 24 hours from the UV-B injury, hence the data presented here were obtained at 24 and 48 hours poststimulation (Fig. 3B). Gelatin SDS-PAGE analysis in samples incubated for 24 hours suggested several MMP candidates (MMP-2, MMP-9, and complex form of the gelatinases with TIMPs) that were, thereafter, individually evaluated using ELISA or Western blot analysis (Fig. 3A). 
A

B
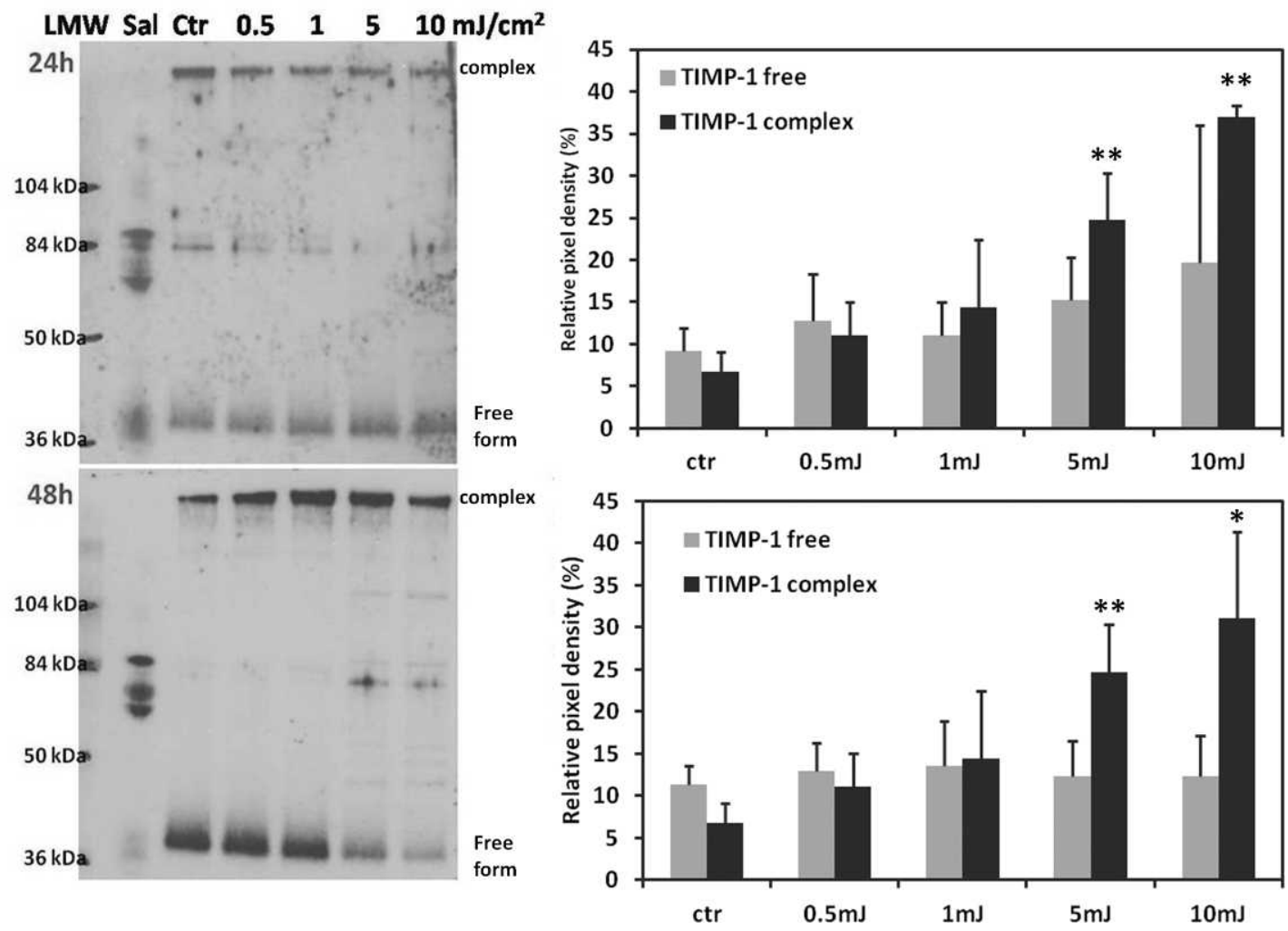

Figure 7. Free and complex form of TIMP-1 in HCE media. (A) Representative Western blot image; (B) densitometry analysis. Equal amounts of media were loaded on the $4 \%$ to $12 \%$ Bis-Tris NuPAGE gel. Data are presented as relative densitometry units normalized to cell lysate protein concentration. ${ }^{*} P<0.05$ compared to control; ${ }^{* *} P<0.01$ compared to control.

ELISA assay for pro-MMP-2 and MMP-2 in complexes showed that untreated HCE cells secreted approximately 4 $\mathrm{ng} / \mathrm{mg}$ of protein MMP-2 at 24 hours, and the secretion increased 2.5 times after 48 hours. In the UV-B-exposed samples, MMP-2 media levels increased with the higher UV-B energy levels, and the increase reached significant values after 48 hours (Fig. 4).

MMP-9 proved to be the main gelatinase in the HCE media. Active and pro-forms of the protein were detected using Western blotting in media conditioned by nontreated and UV-B-treated cells at 24 hours, and the levels increased with the increase in the UV-B energy. At 48 hours, MMP-9 was easily detectable only in the 5 and $10 \mathrm{~mJ} / \mathrm{cm}^{2} \mathrm{UV}$-B-exposed samples in both active and complex form (Figs. 5A, 5B). Strong immunoreactivity at low molecular weights was seen, suggesting that some of the MMP-9 had been degraded. These results were confirmed by the ELISA measurement of pro-MMP-9 (Fig. 5C).

Polymorphonuclear neutrophil-type (PMN) pro-MMP-8 (75-80-kDa molecular form) was barely detectable at 48 hours, and only in media from 5 and $10 \mathrm{~mJ} / \mathrm{cm}^{2}$ treated HCE cells. Interestingly, the same HCE media samples also expressed mesenchymal type of pro and active forms of MMP-8. Relatively strong immunoreactivity was seen in MMP-8 complex forms (Fig. 6).

The high increase in MMP-9 secretion made information on TIMPs imperative; hence, immunodetection at 24 hours poststimulation showed an increase of both free and complex forms of TIMP-1 in the high-energy exposed samples. Intriguingly, at 48 hours TIMP-1 free form levels were uniform in all samples and the complex form was concurrently increasing (Figs. 7A, 7B).

\section{Cytokine Analysis after UV-B Irradiation of HCE Cells}

Since patient tear samples showed significant increase in some secreted cytokines and chemokines, the authors also assessed the contribution of the corneal epithelium to this proinflammatory response. HCE cells exposed to UV-B $(10 \mathrm{~mJ} /$ $\mathrm{cm}^{2}$ ) were compared to nonstimulated cells, in regard to the production of cytokines. From the panel of 36, the authors could detect relative levels of 10 different cytokines or their modulators (Fig. 8). IL-6, IL-8, IL-16, and macrophage migration inhibition factor were positively modulated by UV-B stress, while plasminogen activator inhibitor 1 (PAI-1) secretion (Serpin E1) from stimulated cells decreased more than 2-fold. IL-1 $\beta$ was not detected in unexposed or UV-B-exposed HCE cells.

\section{Discussion}

Advancing age, exposure to environmental UV radiation, and repetitive corneal microtraumas are believed to play significant roles in the pathology of CDK. ${ }^{9-11}$ So far, no mechanistic link between UV exposure and CDK has been established. Here, the authors have addressed this question by analyzing corneal specimens obtained from CDK affected eyes. They collected tear samples from CDK patients and unaffected individuals from the Patagonia region and measured MMP-2 and MMP-9 as well as cytokine levels. Finally, based on these findings, they hypothesized that these proteins are produced by the corneal epithelium, and showed that UV-B induces a pro-inflammatory response in these cells with concomitant increase in MMP-2 and MMP-9 levels. This is then likely to increase the apoptotic/ 


\begin{tabular}{|c|c|c|c|c|c|c|c|c|c|c|}
\hline & 1 & 2 & 3 & 4 & 5 & 6 & 7 & 8 & 9 & 10 \\
\hline A & $\operatorname{ctr}(+)$ & $\mathrm{C5} / \mathrm{C5} \mathrm{a}$ & CD40L & G-CSF & GM-CSF & GRO $\alpha$ & $1-309$ & SICAM-1 & IFN- $\nu$ & $\operatorname{Ctr}(+)$ \\
\hline B & & IL-1 $\alpha$ & IL-1 $\beta$ & IL-1ra & IL-2 & IL-4 & IL-5 & IL-6 & IL-8 & \\
\hline C & & IL-10 & IL-12p & IL-13 & IL-16 & IL-17 & IL-17E & IL-23 & IL-27 & \\
\hline D & & IL-32 $\alpha$ & IP-10 & I-TAC & MCP-1 & MIF & MIP-1 $\alpha$ & MIP-1 $\beta$ & PAI-1 & \\
\hline E & Ctr (+) & RANTES & SDF-1 & TNF- $\alpha$ & STREM-1 & & & & & $\operatorname{tr}(-)$ \\
\hline
\end{tabular}
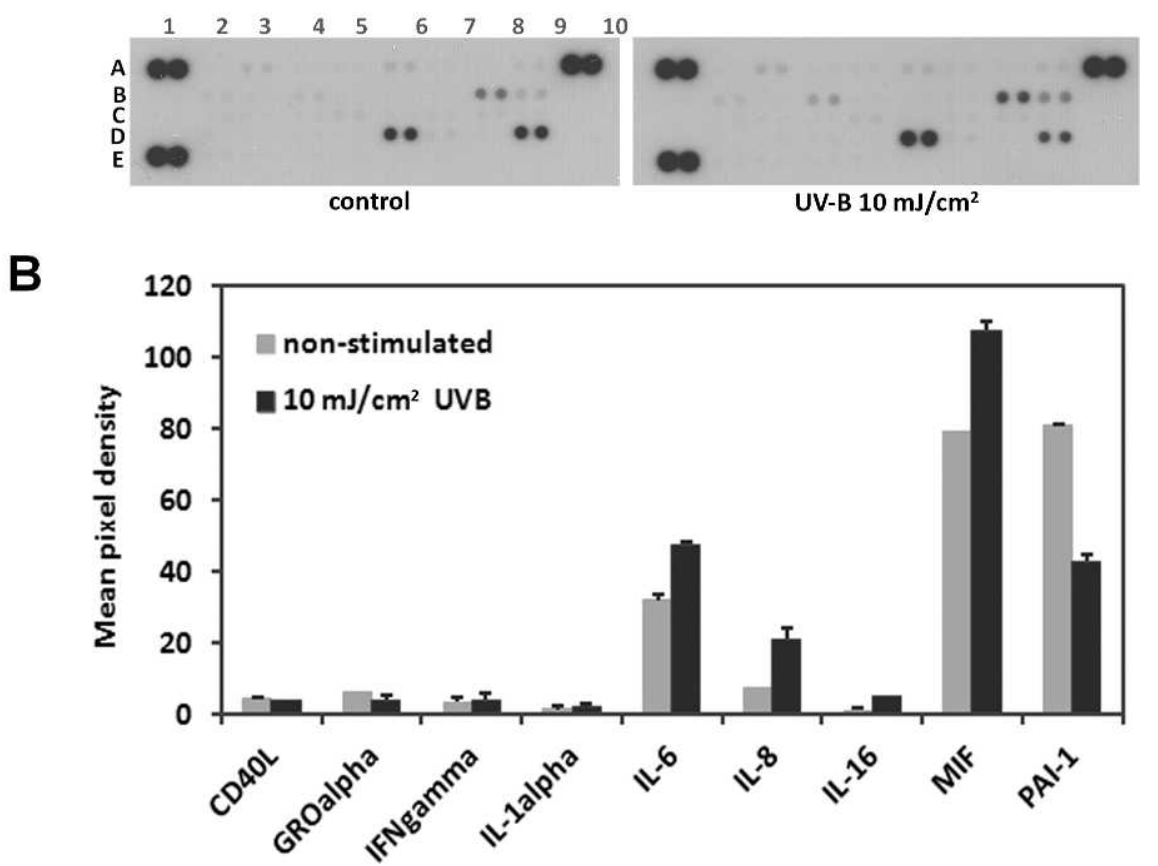

Figure 8. Inflammatory profile of UV-stressed HCE cells. (A) Cytokine array of supernatants from cells exposed to $10 \mathrm{~mJ} / \mathrm{cm}^{2} \mathrm{UV}-\mathrm{B}$ compared to nonstressed cells (control). $700 \mu \mathrm{L}$ of cell conditioned media was prepared as described in the material and methods section and incubated overnight with the antibody spotted nitrocellulose arrays. (B) Mean pixel density measurements for detected cytokines.

necrotic response of the corneal epithelial cells, harm the integrity of the basement membrane, and, eventually, lead to visible changes in the corneal architecture.

The integrity of the corneal epithelium depends largely on the underlying basement membrane, which is predominantly composed of type- 4 collagen and laminin, ${ }^{12,13}$ sensitive to protease activity. MMPs have been implicated in the pathogenesis of several ocular surfaces and tear film disorders, but also maintain and repair corneal architecture. MMP-2 and MMP9 readily digest gelatins and denatured collagens, and participate in corneal wound healing, and in the pathogenesis of inflammatory diseases of the cornea. ${ }^{14-18}$ This study shows that corneas from CDK patients have significant induction of MMP-9 expression, in keeping with the hypothesis that these enzymes play a role in the course of CDK by jeopardizing the integrity of the corneal basement membrane. MMP-2 highlighted the edges of the CDK deposits, even though the staining was detected in both healthy and affected corneas. Using an animal corneal injury model, Fini and coworkers ${ }^{19}$ showed that MMP-9 participated in the degradation of the basement membrane, whereas MMP-2 was involved in the remodeling of the ECM and basement membrane. ${ }^{14,19}$ Accordingly, in diseases where the health of the cornea is jeopardized, it would be expected that MMP-9 secretion would be increased. This increase may either be a balanced response to injury, or an unbalanced response leading to tissue destruction as seems to be the case in CDK.
Previous reports show that diseases, in which the integrity of the corneal epithelium is compromised, were associated with high levels of MMP-9 and MMP-2 in tear fluid. ${ }^{17,20-25}$ Here, it was found that CDK patients have significantly increased concentrations of tear fluid MMP-2 and MMP-9, although active enzyme species were not found. Considering the presence of these enzymes, in particular in the basal cells and in the basement membrane area, it is possible that the high avidity of these MMPs has led to tight binding to their tissue substrates, which is supported by the immunohistochemical staining and the damage in this area. This, together with the tear fluid TIMP, could explain the lack of active MMP-2 and MMP-9 species in zymographies. Additionally, increased levels of gelatinases were seen as well in UV-B-stimulated HCE cell culture supernatants, pointing out that the likely source for these enzymes in tear fluid is the corneal epithelium. Thus, this self-perpetuating system will eventually harm the epithelium and the subepithelial tissues if the enzyme activity cannot be regulated. As the authors' earlier studies show, in CDK, the tight regulation of the MMP/TIMP ratio seems to be lost. ${ }^{4}$

Another finding worth noting was the net inflammatory profile of the CDK ocular surface, suggesting that the epithelium may elicit and maintain a pro-inflammatory cascade making the cornea prone for the development of corneal diseases. The initiating stimulus could be UV radiation, as cornea absorbs all UV-C and most of the UV-B wavelengths in order to protect the structures inside the eye from radiationinduced damage. ${ }^{26}$ Still, corneal epithelial cells are sensitive to 
low wavelength UV, and this stress eventually leads to cell death by necrosis or apoptosis. Renewal of the corneal epithelium is a continuous process that is vital for both the barrier and visual function of the cornea. A plausible hypothesis would be that an overstressed epithelium is promoting the renewal process, rather than protecting the affected cells from death. UV-challenged cells cope with the induced stress using the MMPs in a controlled manner, where overproduction of the enzymes tends to be balanced by the production of TIMPs. However, prolonged stress disrupts this balance with a damaging increase in MMP-9 secretion, unmatched by TIMP-1 production.

In vitro studies demonstrated that UV radiation activates stress signaling pathways leading to increased cytokine production in the ocular surface cells, as well as increased cytokine release into tears. ${ }^{27-29}$ These inflammatory mediators partake in the recruitment of immune cells at the eye surface, and in the subsequent damage caused by the inflammatory reaction. ${ }^{30,31}$ Since CDK patients also presented significantly increased production of cytokines IL- $1 \beta$ and IL-8, it is possible that these contributed to the secretion of MMP-9 and MMP-8, respectively. The cellular model confirmed the corneal epithelium as a significant source of IL-8 and a potential source of IL- 6 . The cytokine response became significant as early as 4 hours after injury, while gelatinases could be measured in the supernatant only after 24 hours. Moreover, UV-B downregulated PAI-1 can contribute to the increased urokinaseplasmin activation of MMPs. ${ }^{32}$ Additional support to the cytokines-MMPs interrelation is brought by the IL-8 regulation of MMP-9 secretion from neutrophils, ${ }^{33}$ while, in turn, MMP-9 can sustain the inflammatory condition by cleaving the secreted IL- 8 to form a more aggressive chemokine. ${ }^{34}$

Therefore, these data suggest that the CDK pathology is underlined by a significant inflammatory response, and that corneal epithelial cells, as the first line of defense, play important roles in this process. The sustained inflammatory condition in turn favors the MMPs-TIMPs dysregulation and its degradative consequences.

\section{References}

1. Urrets-Zavalia JA, Knoll EG, Maccio JP, Urrets-Zavalia EA, Saad JA, Serra HM. Climatic droplet keratopathy in the Argentine Patagonia. Am J Ophthalmol. 2006;141:744-746.

2. Menegay M, Lee D, Tabbara KF, et al. Proteomic analysis of climatic keratopathy droplets. Invest Ophthalmol Vis Sci. 2008;49:2829-2837.

3. Sivak JM, Fini ME. MMPs in the eye: emerging roles for matrix metalloproteinases in ocular physiology. Prog Retin Eye Res. 2002;21:1-14.

4. Holopainen JM, Serra HM, Sanchez MC, et al. Altered expression of matrix metalloproteinases and their tissue inhibitors as possible contributors to corneal droplet formation in climatic droplet keratopathy. Acta Ophthalmol. 2011; 89:569-574.

5. Urrets-Zavalia JA, Maccio JP, Knoll EG, Cafaro T, Urrets-Zavalia EA, Serra HM. Surface alterations, corneal hypoesthesia, and iris atrophy in patients with climatic droplet keratopathy. Cornea. 2007;26:800-804.

6. Sorsa T, Salo T, Koivunen E, et al. Activation of type IV procollagenases by human tumor-associated trypsin-2. J Biol Chem. 1997;272:21067-21074.

7. Ylipalosaari M, Thomas GJ, Nystrom M, et al. Alpha v beta 6 integrin down-regulates the MMP-13 expression in oral squamous cell carcinoma cells. Exp Cell Res. 2005;309:273283.

8. Hsu SM, Raine L, Fanger H. Use of avidin-biotin-peroxidase complex (ABC) in immunoperoxidase techniques: a compar- ison between $\mathrm{ABC}$ and unlabeled antibody (PAP) procedures. $J$ Histochem Cytochem. 1981;29:577-580.

9. Gray RH, Johnson GJ, Freedman A. Climatic droplet keratopathy. Surv Ophthalmol. 1992;36:241-253.

10. Taylor HR, West SK, Rosenthal FS, Munoz B, Newland HS, Emmett EA. Corneal changes associated with chronic UV irradiation. Arch Ophthalmol. 1989;107:1481-1484.

11. Rodger FC, Cuthill JA, Fydelor PJ, Lenham AP. Ultra-violet radiation as a possible cause of corneal degenerative changes under certain physiographic conditions. Acta Ophthalmol (Copenh). 1974;52:777-785.

12. Labermeier U, Kenney MC. The presence of EC collagen and type IV collagen in bovine Descemet's membranes. Biochem Biophys Res Commun. 1983;116:619-625.

13. Millin JA, Golub BM, Foster CS. Human basement membrane components of keratoconus and normal corneas. Invest Ophthalmol Vis Sci. 1986;27:604-607.

14. Matsubara M, Girard MT, Kublin CL, Cintron C, Fini ME. Differential roles for two gelatinolytic enzymes of the matrix metalloproteinase family in the remodelling cornea. Dev Biol. 1991;147:425-439.

15. Kervick GN, Pflugfelder SC, Haimovici R, Brown H, Tozman E, Yee R. Paracentral rheumatoid corneal ulceration. Clinical features and cyclosporine therapy. Ophthalmology. 1992;99: 80-88.

16. Azar DT, Hahn TW, Jain S, Yeh YC, Stetler-Stevensen WG. Matrix metalloproteinases are expressed during wound healing after excimer laser keratectomy. Cornea. 1996;15: 18-24.

17. Afonso AA, Sobrin L, Monroy DC, Selzer M, Lokeshwar B, Pflugfelder SC. Tear fluid gelatinase B activity correlates with IL-1alpha concentration and fluorescein clearance in ocular rosacea. Invest Ophthalmol Vis Sci. 1999;40:2506-2512.

18. Pflugfelder SC, Farley W, Luo L, et al. Matrix metalloproteinase9 knockout confers resistance to corneal epithelial barrier disruption in experimental dry eye. Am J Pathol. 2005;166: 61-71.

19. Fini ME, Parks WC, Rinehart WB, et al. Role of matrix metalloproteinases in failure to re-epithelialize after corneal injury. Am J Pathol. 1996;149:1287-1302.

20. Smith VA, Hoh HB, Easty DL. Role of ocular matrix metalloproteinases in peripheral ulcerative keratitis. $\mathrm{Br} J$ Ophthalmol. 1999;83:1376-1383.

21. Sobrin L, Liu Z, Monroy DC, et al. Regulation of MMP-9 activity in human tear fluid and corneal epithelial culture supernatant. Invest Ophthalmol Vis Sci. 2000;41:1703-1709.

22. Solomon A, Li DQ, Lee SB, Tseng SC. Regulation of collagenase, stromelysin, and urokinase-type plasminogen activator in primary pterygium body fibroblasts by inflammatory cytokines. Invest Ophthalmol Vis Sci. 2000;41:21542163.

23. Smith VA, Rishmawi H, Hussein H, Easty DL. Tear film MMP accumulation and corneal disease. Br J Ophthalmol. 2001;85: 147-153.

24. Kumagai N, Yamamoto K, Fukuda K, et al. Active matrix metalloproteinases in the tear fluid of individuals with vernal keratoconjunctivitis. J Allergy Clin Immunol. 2002;110:489491.

25. Sakimoto T, Shoji J, Kanno H, Sawa M. Gelatinase expression in ocular surface disorders. Jpn J Ophthalmol. 2004;48:17-22.

26. Lu L, Reinach PS, Kao WW. Corneal epithelial wound healing. Exp Biol Med (Maywood). 2001;226:653-664.

27. Yoshizumi M, Nakamura T, Kato M, et al. Release of cytokines/ chemokines and cell death in UVB-irradiated human keratinocytes, HaCaT. Cell Biol Int. 2008;32:1405-1411.

28. Black AT, Gordon MK, Heck DE, Gallo MA, Laskin DL, Laskin JD. UVB light regulates expression of antioxidants and 
inflammatory mediators in human corneal epithelial cells. Biochem Pharmacol. 2011;81:873-880.

29. Acera A, Rocha G, Vecino E, Lema I, Duran JA. Inflammatory markers in the tears of patients with ocular surface disease. Ophthalmic Res. 2008;40:315-321.

30. Thakur A, Willcox MD, Stapleton F. The proinflammatory cytokines and arachidonic acid metabolites in human overnight tears: homeostatic mechanisms. J Clin Immunol. 1998; 18:61-70.

31. Sack RA, Beaton A, Sathe S, Morris C, Willcox M, Bogart B. Towards a closed eye model of the pre-ocular tear layer. Prog Retin Eye Res. 2000;19:649-668.
32. Mazzieri R, Masiero L, Zanetta L, et al. Control of type IV collagenase activity by components of the urokinase-plasmin system: a regulatory mechanism with cell-bound reactants. EMBO J. 1997;16:2319-2332.

33. Chakrabarti S, Patel KD. Regulation of matrix metalloproteinase-9 release from IL-8-stimulated human neutrophils. $J$ Leukoc Biol. 2005;78:279-288.

34. Van den Steen PE, Proost P, Wuyts A, Van Damme J, Opdenakker G. Neutrophil gelatinase B potentiates interleukin-8 tenfold by aminoterminal processing, whereas it degrades CTAP-III, PF- 4 , and GRO-alpha and leaves RANTES and MCP-2 intact. Blood. 2000;96:2673-2681. 